\title{
Large-eddy simulation of plant canopy flows using plant-scale representation
}

\author{
Wusi Yue - Marc B. Parlange - Charles Meneveau • \\ Weihong Zhu • René van Hout • Joseph Katz
}

Received: 25 April 2006 / Accepted: 23 February 2007 / Published online: 30 March 2007

(C) Springer Science+Business Media B.V. 2007

\begin{abstract}
Turbulent flow in a corn canopy is simulated using large-eddy simulation (LES) with a Lagrangian dynamic Smagorinsky model. A new numerical representation of plant canopies is presented that resolves approximately the local structure of plants and takes into account their spatial arrangement. As a validation, computational results are compared with experimental data from recent field particle image velocimetry (PIV) measurements and two previous experimental campaigns. Numerical simulation using the traditional modelling method to represent the canopy (field-scale approach) is also conducted as a comparison to the plant-scale approach. The combination of temporal PIV data, LES and spatial PIV data allows us to couple a wide range of relevant turbulence scales. There is good agreement between experimental data and numerical predictions using the plant-scale approach in terms of various turbulence statistics. Within the canopy, the plant-scale approach also allows the capture of more details than the field-scale approach, including instantaneous gusts that penetrate deep inside the canopy.
\end{abstract}

\footnotetext{
W. Yue

Department of Geography and Environmental Engineering and Center for Environmental and Applied Fluid Mechanics, The Johns Hopkins University, Baltimore, MD 21218, USA e-mail: yue@jhu.edu
}

\section{B. Parlange}

School of Architecture, Civil and Environmental Engineering, Ecole Polytechnique Federale de Lausanne, Lausanne 1015-CH, Switzerland

e-mail: marc.parlange@epfl.ch

\section{Meneveau $(\varangle) \cdot$ W. Zhu · R. van Hout · J. Katz}

Department of Mechanical Engineering and Center for Environmental and Applied Fluid Mechanics, The Johns Hopkins University, Baltimore, MD 21218, USA

e-mail: meneveau@jhu.edu

Present Address:

R. van Hout

Faculty of Mechanical Engineering, Technion - Israel Institute of Technology, Technion City, Haifa 32000, Israel 
Keywords Canopy turbulence · Corn canopy · Energy spectrum · Large-eddy simulation

\section{Introduction}

The interaction between a plant canopy and the atmospheric boundary layer results in complex structures of turbulence, which govern the transport of kinetic energy and momentum, as well as the transport of scalars including water vapour, heat, carbon dioxide, pollen, and disease spores. The understanding of turbulent transport processes at the plant-atmosphere interface is important for fields ranging from canopy physiology and agricultural micrometeorology to ecological studies of genetically engineered plants (Wolfenbarger and Phifer 2000). Canopy turbulence is characterized by the absorption of momentum by aerodynamic foliage drag throughout the whole canopy depth, which leads to an unstable inflected velocity profile, similar to that in a mixing layer. Dissipation of turbulent kinetic energy (TKE) inside the canopy is enhanced by means of wake-scale eddies (Finnigan 2000). To date, the complexity of turbulence structures and high turbulence intensities inside canopies have prevented the development of a general similarity scheme in canopy turbulence like, for example, the Monin-Obukhov theory in the atmospheric surface layer. Interests in quantifying turbulence statistics and exploring large-scale coherent structures in plant canopies have led to a number of experimental investigations for different types of canopies, such as forests (Shaw et al. 1988; Gardiner 1994), cereal crops (Shaw et al. 1974; Wilson et al. 1982; van Hout et al. 2007), and wind-tunnel canopy models (Seginer et al. 1976; Brunet et al. 1994; Zhu et al. 2006), to name just a few. Some important turbulence structures have been observed in the experiments, e.g., coherent scalar ramp patterns (Gao et al. 1989) and wavelike travelling disturbances in cereal canopies (Finnigan 1979). For more comprehensive reviews on this subject the reader is referred to the papers by Raupach and Thom (1981) and Finnigan (2000).

High turbulence intermittency observed in canopy flows raises difficulties in numerical simulations based on the Reynolds-averaged (ensemble-averaged) Navier-Stokes equations. Because of the increasing computer power, large-eddy simulation (LES) has gained popularity in numerical modelling of canopy flows since the pioneering work of Shaw and Schumann (1992). In LES, canopies are usually treated as a porous body of horizontally uniform area density. Since the Shaw and Schumann study a number of LES studies of plant canopy turbulence have been performed including Kanda and Hino (1994) who examined organized structures in a developing flow over a plant canopy; Dwyer et al. (1997) calculated the turbulent kinetic energy budget above and within a forest; Shen and Leclerc (1997) compared turbulence statistics and structures under neutral and unstable conditions; and Patton et al. (1998) simulated a windtunnel wheat canopy with a windbreak. Most of these simulations imposed a mean pressure-gradient driving the fully developed turbulent flows. Su et al. (1998) added an artificial momentum source at the top boundary of the computational domain as the driving force of the flow to address the issue of non-constant momentum flux layer above the canopy predicted by all other LES studies. More recently, Shaw and Patton (2003) studied the wake effects on TKE transfer processes and found that the wake energy is comparable to the subgrid-scale (SGS) energy inside the canopy, thus providing a "short-circuit" for the inertial energy cascade process. Fitzmaurice et al. (2004) examined scalar microfronts using conditional sampling and compositional 
technique. Watanabe (2004) used a wavelet transform to detect a scalar's microfrontal structure in the scalar time trace under different flow conditions (both shear-driven and pressure-driven flows).

Most of the above-mentioned LES studies modelled the canopies as a horizontally homogenous drag field that we refer to as the "field-scale" approach. The heterogeneity of the canopies, characterized by local structural features of plants ("plant-scale") and field arrangement, has not been taken into account. Furthermore, in these simulations, the SGS models rely on the standard Smagorinsky parameterization (Shaw and Schumann 1992; Moeng 1984), whereas the appropriate Smagorinsky coefficient may critically depend on local flow conditions inside the canopy. To date, LES studies of canopy flows have used an a priori prescribed coefficient that typically does not depend on the local flow structures. Addressing these issues, our study presents a flexible approach that takes into account the spatial structure of a corn canopy and uses a newly developed scale-dependent Lagrangian dynamic SGS model that does not require a priori specification of the model coefficient (Bou-Zeid et al. 2005). The computational results are compared with those from our recent particle image velocimetry (PIV) field experiment and two other previous sets of measurements in corn fields from the literature. The PIV measurements were carried out in a half-circular corn field of $0.6 \mathrm{~km}^{2}$ located on the eastern shore of the Chesapeake Bay, Maryland, USA, in July 2003. The PIV instrumentation was mounted on a retractable measurement platform that could be rotated to align the sample area $(182 \mathrm{~mm} \times 182 \mathrm{~mm})$ with the governing wind direction. The measurements were performed at four different elevations, from just below the canopy top up to $1 \mathrm{~m}$ above it. At each elevation, 4096 double-exposure PIV images were recorded at an acquisition rate of $4 \mathrm{~Hz}$, corresponding to $1,024 \mathrm{~s}$ of sampling time. Before each run, the laser sheet was aligned with the wind direction using a wind vane mounted on top of the platform. In addition, a meteorological tower was set up composed of two three-dimensional sonic anemometers (Campbell Scientific) exactly at the canopy top, $z / h=1$, and the other at $z / h=1.35$, where $z$ is the surface normal coordinate measured from the ground up and $h=2.67 \mathrm{~m}$ is the canopy top. Wind data was continuously acquired at $6.9 \mathrm{~Hz}$. Measurement data used here were acquired on July 22, 2003, between 0030 and 0300 Eastern Daylight Time (EDT). For additional details on the field experiment, the reader is referred to van Hout et al. (2007).

Two sets of existing experimental data from corn canopies (Shaw et al. 1974; Wilson et al. 1982) are also used in the assessment of the present LES results. These data were collected at the Elora Research Station, Elora, Ontario, Canada, over different years. The corn plants were $760 \mathrm{~mm}$ apart. The measurements for the "Shaw" corn field study (with a leaf area index (LAI) of 3.0 and plant height $2.90 \mathrm{~m}$ ) were taken from 1400 to 1600 EDT on October 5, 1971. For the "Wilson" corn field (with LAI 2.9), the experiments were carried out between September 13 and October 5 in 1976 and between July 28 and September 3 in 1977. The averaged canopy height was $2.25 \mathrm{~m}$ during the experiments. Turbulence statistics, such as root-mean-square velocity and momentum flux, and energy spectra were obtained with a split-film anemometer in both experiments.

The objective of this paper is to test two different representations of plant canopy (averaged "field-scale" and detailed "plant-scale") and compare the simulation results, e.g. mean flow and turbulence statistics, with the experimental data. A brief description of the LES and the Lagrangian SGS model is given in Sect. 2. The numerical modelling of the corn canopy is described in the same section. In Sect. 3 the 
computational turbulence statistics and energy spectra are compared with the experimental data. Section 4 provides the summary and conclusions of this work.

\section{Numerical Modelling}

\subsection{Large-eddy simulation}

In LES, energy-containing large-scale eddies are computationally resolved, while small-scale eddies, tending to be more homogeneous and isotropic, are modelled. The large scales are separated from the small scales via a filtering process, thus a variable is decomposed into a resolved scale component and an unresolved SGS component. The filtered, incompressible continuity and Navier-Stokes equations are,

$$
\begin{array}{r}
\frac{\partial \tilde{u}_{i}}{\partial x_{i}}=0, \\
\frac{\partial \tilde{u}_{i}}{\partial t}+\tilde{u}_{j}\left(\frac{\partial \tilde{u}_{i}}{\partial x_{j}}-\frac{\partial \tilde{u}_{j}}{\partial x_{i}}\right)=-\frac{\partial \tilde{p}^{*}}{\partial x_{i}}-\frac{\partial \tau_{i j}}{\partial x_{j}}+F_{i},
\end{array}
$$

where represents filtering at the grid scale; $\tilde{u}_{i}$ is the filtered velocity component, $i=1,2,3$ corresponding to the $x, y, z$ directions (see Fig. 1). The spectral cutoff filter is used in the present LES. The convective terms are written in the rotational form for better numerical conservation of kinetic energy. The viscous diffusion term is neglected because of the very high Reynolds number in atmospheric flows and modelling of the surface layer near the ground. $F_{i}$ represents external forces. The modified pressure $\tilde{p}^{*}$ is defined as

$$
\tilde{p}^{*}=\tilde{p} / \rho+\tilde{u}_{k} \tilde{u}_{k} / 2+\left(\widetilde{u_{k} u_{k}}-\tilde{u}_{k} \tilde{u}_{k}\right) / 3
$$

where the repeated subscript $k$ implies summation. The traceless part of the SGS stress $\tau_{i j}$, introduced by the filtering of the non-linear convective terms, is defined as

$$
\tau_{i j}=\widetilde{u_{i} u_{j}}-\tilde{u}_{i} \tilde{u}_{j}-\left(\widetilde{u_{k} u_{k}}-\tilde{u}_{k} \tilde{u}_{k}\right) \delta_{i j} / 3 .
$$

The SGS stress represents the effects of small-scale eddies filtered out from the resolved large-scale eddies. An important role of eddies is to transfer energy from large scales to smaller scales in a cascade process until the energy is dissipated at the Kolmogorov scale by molecular viscosity. Since the SGS stress is not computable directly, an SGS model is used to express $\tau_{i j}$ in terms of the resolved-scale velocity field. The success of LES relies on the capability of the SGS model to accurately

Fig. 1 Schematic of arrangement of the corn field (not to scale)

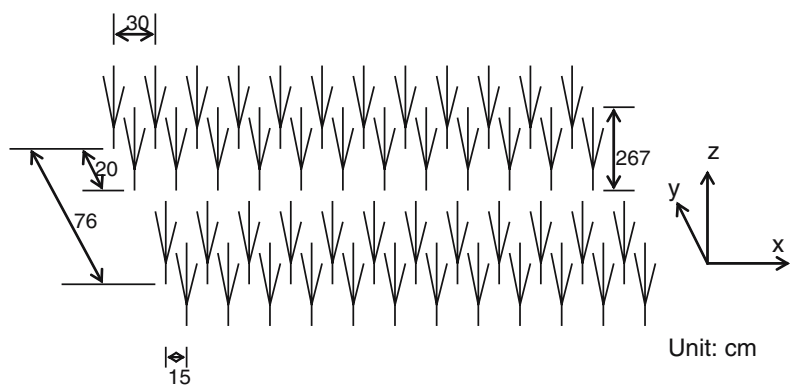


compute the SGS effects based on the resolved scales. Many SGS models used in the lower atmosphere are based on the Smagorinsky model,

$$
\tau_{i j}=-2\left(C_{s} \Delta\right)^{2}|\tilde{S}| \tilde{S}_{i j}
$$

where $\Delta$ is a length scale defined as $\Delta=\sqrt[3]{\Delta x \Delta y \Delta z} . \tilde{S}_{i j}$ is the resolved strain rate, $\tilde{S}_{i j}=\left(\partial \tilde{u}_{i} / \partial x_{j}+\partial \tilde{u}_{j} / \partial x_{i}\right) / 2$, and $\tilde{S}=\sqrt{2 \tilde{S}_{i j} \tilde{S}_{i j}}$. The Smagorinsky coefficient, $C_{s}^{2}$, is a parameter that typically varies for different flows. Germano's dynamic procedure (Germano et al. 1991; Lilly 1992) for obtaining $C_{s}^{2}$ has become a popular approach in SGS modelling. This procedure makes use of a second larger-scale filter, $\widehat{ }$, and an algebraic identity, the Germano identity, between the SGS stresses from the two filters. An analytical expression can be developed for $C_{s}^{2}$ that usually needs to be averaged over directions of statistical homogeneity to stabilize computations. But this approach may not be applicable to turbulent flows over complex surfaces (i.e., plants) in which there is no appreciable homogeneous flow direction. Meneveau et al. (1996) developed a Lagrangian procedure to average $C_{s}^{2}$ over fluid-particle trajectories, which does not rely on specific flow conditions thus relaxing the requirement for homogeneous flow directions and making the model particularly well suited for turbulent flows in complex configurations. An assumption in the dynamic procedure described above is that $C_{s}^{2}$ is independent of filter scale, so-called scale-invariance. This assumption may break down when the filter scale $\Delta$ approaches the integral scale, e.g., in the vicinity of a wall surface (Meneveau and Katz 2000). Porté-Agel et al. (2000) developed a scale-dependent dynamic model in which $C_{s}^{2}$ is a function of filter size, employing the same averaging procedures as in Germano et al. (1991). A filter at a larger scale, $4 \Delta\left(\right.$ denoted as $\sim$ ), is employed to determine $\beta=C_{s, 2 \Delta}^{2} / C_{s, \Delta}^{2}$, that is a parameter accounting for possible scale dependence of $C_{s, \Delta}^{2}$. The parameter $\beta$ is assumed to be scale invariant, i.e., $\beta=C_{s, 2 \Delta}^{2} / C_{s, \Delta}^{2}=C_{s, 4 \Delta}^{2} / C_{s, 2 \Delta}^{2}$. Bou-Zeid et al. (2005) recently developed a scale-dependent Lagrangian dynamic model for complex turbulent flows that combines the scale-dependent model of Porté-Agel et al. (2000) and the Lagrangian averaging procedure of Meneveau et al. (1996). In this model, it is shown that $C_{s, \Delta}^{2}$ at the grid scale can be calculated by

$$
C_{s, \Delta}^{2}=\frac{\mathcal{I}_{L M} / \mathcal{I}_{M M}}{\max \left(\frac{\mathcal{I}_{Q N} \mathcal{I}_{M M}}{\mathcal{I}_{N N} \mathcal{I}_{L M}}, 1 / 8\right)}
$$

where

$$
\mathcal{I}_{A B}=\int_{-\infty}^{t} A_{i j} B_{i j}\left[\mathbf{x}\left(t^{\prime}\right), t^{\prime}\right] W\left(t-t^{\prime}\right) \mathrm{d} t^{\prime} .
$$

Here, $W(t)$ is an exponential weighting function, $W(t)=e^{-t / T} / T$, and $T$ is a time scale, chosen as $T=1.5 \Delta\left(\mathcal{I}_{L M} \mathcal{I}_{M M}\right)^{-1 / 8}$ based on direct numerical simulation data and self-consistency (Meneveau et al. 1996). In addition, $\mathbf{x}\left(t^{\prime}\right)$ is the location of fluid elements at a prior time $t^{\prime}, \mathbf{A}$ and $\mathbf{B}$ represent tensors $(\mathbf{L}, \mathbf{M})$ and $(\mathbf{N}, \mathbf{Q})$, respectively, computed as follows, 


$$
\begin{aligned}
& L_{i j}=\widehat{\tilde{u}}_{i} \tilde{u}_{j}-\hat{\tilde{u}}_{i} \hat{\tilde{u}}_{j}, \\
& M_{i j}=2 \Delta^{2}\left[{\widehat{\tilde{S}} \mid \tilde{S}_{i j}}-4 \beta|\hat{\tilde{S}}| \hat{\tilde{S}}_{i j}\right] \text {, } \\
& Q_{i j}=\overbrace{\tilde{u}_{i} \tilde{u}_{j}}-\overbrace{\tilde{u}_{i}} \overbrace{\tilde{u}_{j}}, \\
& N_{i j}=2 \Delta^{2}[\overbrace{|\tilde{S}| \tilde{S}_{i j}}-16 \beta|\overbrace{\tilde{S}}| \overbrace{\tilde{S}_{i j}}] .
\end{aligned}
$$

More detailed formulation and algorithmic aspects of the Lagrangian dynamic SGS model can be found in Meneveau et al. (1996) and Bou-Zeid et al. (2005).

The LES equations (2) are solved by applying a pseudo-spectral method in the horizontal directions and centered finite-difference method in the vertical direction (Albertson and Parlange 1999a,b; Porté-Agel et al. 2000). The vertical velocity component, $w$, is staggered with horizontal components $u$ and $v$. In the pseudo-spectral method, the spatial derivatives are calculated in spectral space while the nonlinear convective terms are evaluated in physical space for computational efficiency. The convective terms are de-aliased by padding and truncation using the $3 / 2$ rule (Orszag 1970). The second-order Adams-Bashforth scheme is used for time advancement.

\subsection{Numerical representation of corn canopy}

The corn field where the PIV measurements were conducted was double-row planted (see Fig. 1). Between two normal corn plant rows (0.76 m apart), an extra staggered plant row was planted in order to increase corn production. The height of the mature corn plants at the test site was on average $2.67 \mathrm{~m}$. The LAI for this corn field was about 6.0, approximately twice as high as in the corn field of Wilson et al. (1982) which did not have the extra plant row. In order to validate the LES results, we employ the same corn field configuration is employed in our simulations and the simulation results, the same are compared to the PIV experimental data. The averaged wind direction during the measurements was roughly aligned with the plant rows ( $x$-direction in Fig. 1 ); here we set the $x$-direction as the streamwise direction in the simulations.

In previous large-eddy simulations of the canopy flows as reviewed in Sect. 1, the canopies were modelled as a horizontally homogeneously distributed drag (force per unit mass) field. The drag force $d_{i}$ is applied at every grid points below the top of the plants without considering the spatial variability of the canopies (as shown in Fig. 2a).

$$
d_{i}=-C_{d} a(z) \tilde{u}_{i}|\tilde{\mathbf{u}}|
$$

where $C_{d}$ is a prescribed drag coefficient, $a(z)$ is the leaf area density, defined as one-sided projected area of leaves per unit volume of canopy, and $|\tilde{\mathbf{u}}|=\sqrt{\tilde{u}_{j} \tilde{u}_{j}}$.

In this paper, we propose a new numerical approach to model air flows through plant canopies, named "plant-scale" approach. In the plant-scale approach, the spatial arrangement of the plants is approximately resolved and the local morphology (stem and leaf) of the plants is explicitly taken into account, as shown in Fig. 2b in a horizontal cross-section through canopy. In the horizontal cross-section, a single corn plant is represented by a stem point at its centre and eight surrounding leaf points, marked as " 0 " and " $\mathrm{x}$ " in Fig. 2b, respectively. At the leaf grid points, the drag force $d_{i}$, Eq. 12, is applied, while at the stem points, a drag force per unit mass $f_{i}$ is imposed, calculated in terms of drag on a cylinder, 


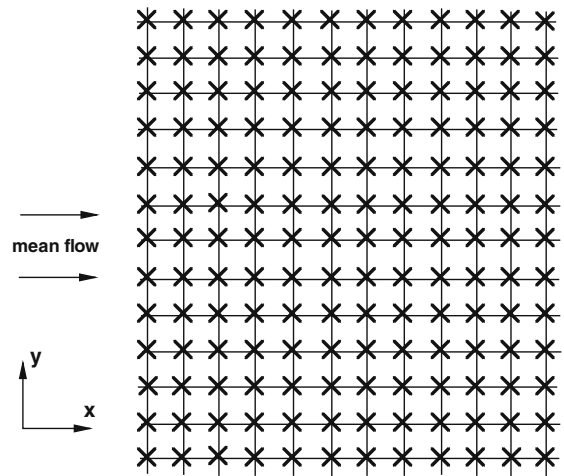

(a) field-scale

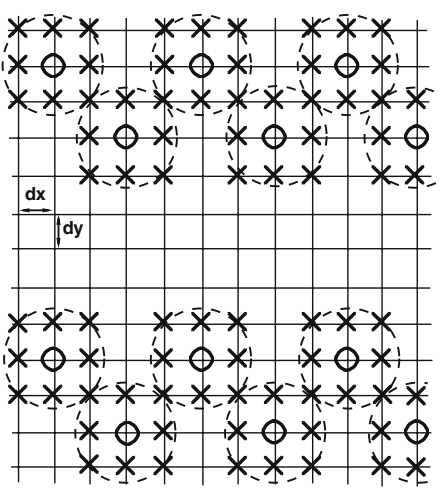

(b) plant-scale

Fig. 2 Schematic of horizontal grid in plant- and field-scale approaches. (a) Field-scale approach; (b) plant-scale approach. Symbols "o" and "x" represent stem and leaf grid points of the corn plants, respectively. The large dashed circle in (b) represents the simulation area for a single corn plant

$$
f_{i}=-\frac{C_{0} D}{2 \mathrm{~d} x \mathrm{~d} y} \tilde{u}_{0, i}\left|\tilde{\mathbf{u}}_{0}\right|,
$$

where $C_{0}$ is the cylinder drag coefficient, around 1.0 for $R e>1,000$ (set to 1.0 in this study for simplicity). $D$ is the diameter of the corn stem (taken independent of $z$ ), and $\tilde{u}_{0, i}$ is the upstream velocity for the cylinder, calculated at a distance $L$ upstream of the stem points (see Fig. 3 ), where $L$ is set equal to the stream-wise grid size $\mathrm{d} x$, which is equal to $3.5 D$ in this study. $\left|\tilde{\mathbf{u}}_{0}\right|$ is the magnitude of the upstream velocity, equal to $\sqrt{\tilde{u}_{0, i} \tilde{u}_{0, i}}$. The upstream velocity is calculated by a trilinear interpolation in terms of the corner point velocity of the grid cell containing the upstream point $P(x, y, z)$ :

$$
\begin{aligned}
& \tilde{u}_{0, i}(x, y, z)=\left(1-s_{1}\right)\left(1-s_{2}\right)\left(1-s_{3}\right) \tilde{u}_{i}^{1}+s_{1}\left(1-s_{2}\right)\left(1-s_{3}\right) \tilde{u}_{i}^{2} \\
& +s_{1} s_{2}\left(1-s_{3}\right) \tilde{u}_{i}^{3}+\left(1-s_{1}\right) s_{2}\left(1-s_{3}\right) \tilde{u}_{i}^{4}+\left(1-s_{1}\right)\left(1-s_{2}\right) s_{3} \tilde{u}_{i}^{5} \\
& +s_{1}\left(1-s_{2}\right) s_{3} \tilde{u}_{i}^{6}+s_{1} s_{2} s_{3} \tilde{u}_{i}^{7}+\left(1-s_{1}\right) s_{2} s_{3} \tilde{u}_{i}^{8}
\end{aligned}
$$

where $s_{1}=(x-x 1) /(x 2-x 1), s_{2}=(y-y 1) /(y 2-y 1)$, and $s_{3}=(z-z 1) /(z 2-z 1)$. $\tilde{u}_{i}^{k}(k=1,2, \ldots, 8)$ is the velocity at the corner points of the interpolation box. The external force $F_{i}$ in Eq. 2 then contains the sum of $d_{i}$ and $f_{i}$. Note that in the "fieldscale" (FS) approach, only the leaf drag force $d_{i}$ is applied at every grid point below the canopy top, assuming homogeneity of the corn canopy. The drag coefficient $C_{d}$ was set to 0.17 as measured by Wilson et al. (1982). The vertical distribution of leaf area density $a(z)$ measured by Wilson et al. (1982) and denoted as $a(z)^{w}$ (see Fig. 4), was modified for the field- and plant-scale approaches. Using the field-scale approach, denoted by superscript "FS", $a(z)^{w}$ is modified by the ratio of LAI's:

$$
a(z)^{F S}=\frac{L A I^{p}}{L A I^{w}} a(z)^{w},
$$

where $L A I^{w}=2.9$ and $L A I^{p}=6.0$ (current field). For the cases using the plant-scale (PS) approaches, $a(z)^{w}$ was also modified by taking into account of area ratio of actual occupied leaf grid cells, i.e.,

$$
a(z)^{P S}=\frac{A_{\text {domain }}}{A_{\text {leaf }}} a(z)^{w},
$$


Fig. 3 Schematic of calculation of upstream velocity $\tilde{\mathbf{u}}_{0}$

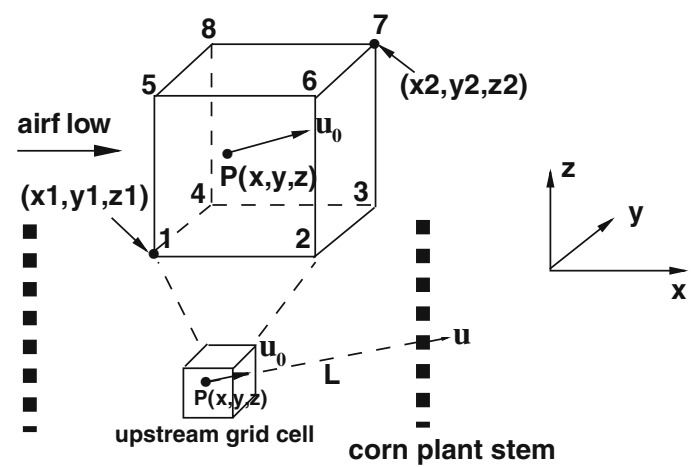

Fig. 4 Leaf area density of corn plants for field-scale approach, $a(z)^{F S}$, normalized by the canopy height $h$

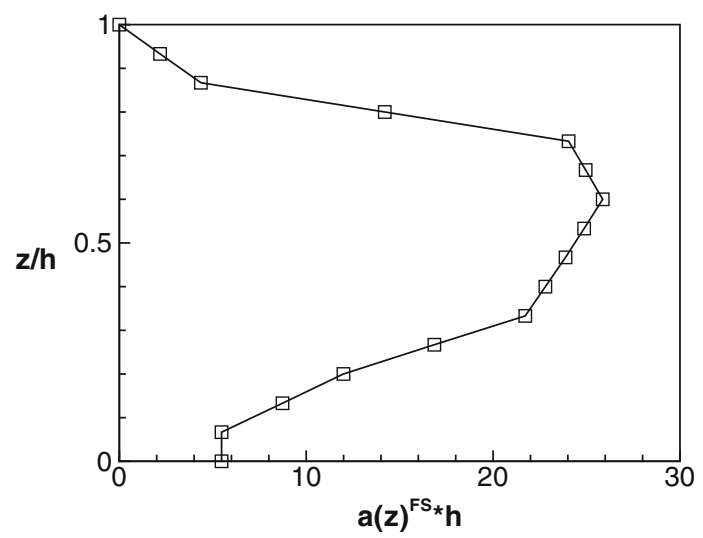

Table 1 Simulation cases

\begin{tabular}{llll}
\hline Cases & Description & $L_{x}: L_{y}: L_{z}$ (domain size) & $N_{x}: N_{y}: N_{z}$ (grid points) \\
\hline PS-1 & plant-scale & $4.5 h: 2.4 h: 3.93 h$ & $160: 64: 60$ \\
PS-2 & plant-scale & $9.0 h: 2.4 h: 3.93 h$ & $320: 64: 60$ \\
FS-1 & field-scale & $24.7 h: 12.3 h: 3.93 h$ & $160: 64: 60$ \\
FS-2 & field-scale & $9.0 h: 2.4 h: 3.93 h$ & $320: 64: 60$ \\
\hline
\end{tabular}

where $A_{\text {domain }}$ is the total horizontal domain area, and $A_{\text {leaf }}$ is the horizontal area occupied by leaf nodes in the plant-scale simulations.

In order to compare plant- and field-scale approaches in terms of simulation results and also investigate the effects of computational domain size and grid resolution, we have conducted four simulation cases, described in Table $1 . L_{x}, L_{y}$, and $L_{z}$ are domain size in the respective, $x$ (streamwise), $y$ (spanwise), and $z$ (vertical) directions, and $N_{x}$, $N_{y}$, and $N_{z}$ are grid points used accordingly in the same directions. The simulation results are compared with experimental data including the PIV measurements as a validation of the simulations. In all cases, the canopy height $h$ and vertical domain size (equal to 3.93h) are fixed. The canopy plants are resolved with 16 uniformly distributed grid points in the vertical direction $z$. PS-2 and FS-2 have the same domain size and same grid points $(320 \times 64 \times 60)$, to compare the two numerical approaches. PS- 2 has a streamwise domain size twice that of PS-1, used as a domain size sensitivity test, while FS-2 has a different grid resolution and horizontal domain size from those of 
FS-1, as a sensitivity test of both grid resolution and domain size. Note that for PS-1 and FS-1, the same grid points (uniformly distributed in each direction), are used but their computational domain sizes are very different (see Table 1). Fully developed turbulent flows are considered, driven by a mean pressure gradient to match the normalized mean flow rate in the experiments. Periodic boundary conditions are employed in the horizontal directions (both $x$ - and $y$-directions) and a shear-free condition is used at the top boundary. Wall stresses at the bottom boundary are specified using the law-of-the-wall. The ground roughness $z_{0}$ is set as $z_{0} / h=0.001$ (a larger value of 0.01 was also tested without showing significant difference in numerical predictions). The time step for each case is varied by setting a CFL number to 0.1 for numerical stability,

$$
\mathrm{d} t=\frac{C F L}{\max _{\Omega}\left(\left|u_{i, j, k}\right| / \mathrm{d} x+\left|v_{i, j, k}\right| / \mathrm{d} y+\left|w_{i, j, k}\right| / \mathrm{d} z\right)}, \quad i, j, k \in \Omega
$$

where the subscript $\Omega$ represents the whole computational domain, and $\mathrm{d} x, \mathrm{~d} y$, and $\mathrm{d} z$ are the grid spacings in the respective coordinate directions.

\section{Results and discussion}

The predicted turbulence statistics and energy spectra by the two numerical approaches (plant- and field-scale), in terms of temporal and spatial (horizontal) average, are compared with available experimental data. The experimental data used here include our recent PIV measurements and previous experiments of Shaw et al. (1974) and Wilson et al. (1982). The normalized and curve-fitted data of Shaw et al. (1974) and Wilson et al. (1982) were extracted from the review paper of Finnigan (2000) and are quoted in this paper. Most figures presented here only show the vertical profiles below $z / h=2$ because the available experimental data are for below this height. Finally, the fluctuating velocity field is examined to investigate specific turbulence structures. Angle brackets $<>$ represent both temporal and horizontal averaging. The superscript ', e.g. in $u^{\prime}$, denotes temporal fluctuation, i.e. deviation from the time-averaged mean value. The superscript ", e.g. in $u^{\prime \prime}$, denotes spatial variation, i.e. deviation from the horizontally-averaged mean value. Good statistical convergence was obtained by averaging over $2 \times 10^{5}$ time-steps and over the horizontal plane at each height. Time convergence was verified by increasing the number of averaging time-steps.

\subsection{Mean streamwise velocity}

Figure 5a shows the mean streamwise velocity profiles, where $\left.<u_{h}\right\rangle$ is the mean streamwise velocity at the canopy top (van Hout et al. 2007). In the PIV profiles, the corresponding $\left\langle u_{h}\right\rangle$ is obtained from the measurements of the sonic anemometers fixed at $z / h=1$. The vertical velocity profiles predicted by both the plant- and field-scale approaches agree well with the experimental data of Wilson et al. (1982) within the canopy and lie between those of PIV and Shaw et al. (1974) above the canopy. Note that we only show the results for PS-1 and FS-1. Considering that in the plant-scale simulation there is a gap between the double rows (see Fig. 2a), the mean velocity profile only averaged over the gap is also plotted and labelled as "PS-1-GA" in the figure and hereafter. As expected the mean velocity in the gap is larger than the domain averaged profile inside the canopy. Deep inside the canopy $(z / h<0.7)$, both 
Fig. 5 Comparison between predicted and measured mean streamwise velocity profiles,

(a) compared with experimental data; (b) in logarithmic vertical coordinate
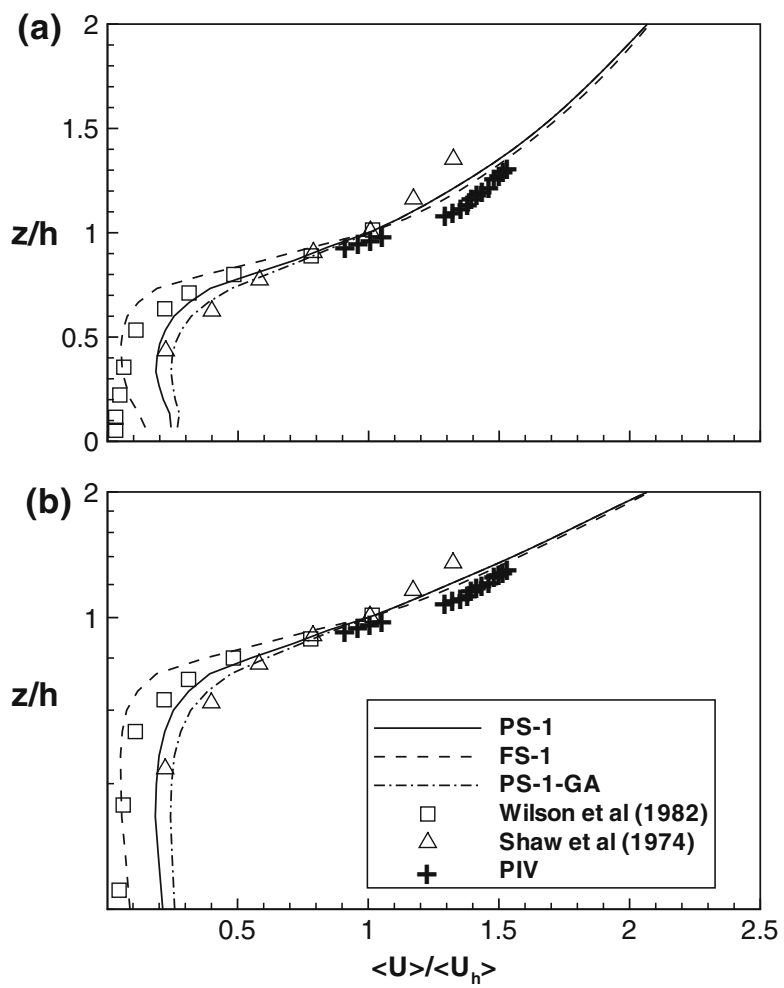

LES and experiments show very steep velocity profiles decreasing rapidly. All the profiles exhibit inflection points (maximum shear) around the canopy top, implying hydrodynamic instability.

In Fig. 5b, the vertical coordinate is plotted in a logarithmic scale. The linear behaviour of the LES results above the canopy top is in accordance with the logarithmic law. These profiles are shifted up with a displacement $d$, called the zero-displacement height, away from the ground, i.e.,

$$
U(z)=\frac{u_{*}}{\kappa} \ln \left(\frac{z-d}{z_{0}}\right)
$$

The determination of $d$, however, is non-trivial. A regression analysis usually results in errors of at least 25\% (Bradley and Finnigan 1973) because of the insensitivity of Eq. 18 involving a fit of three parameters, $u_{*}, d$, and $z_{0}$. Since $d$ can also be interpreted as the mean level of momentum absorption by the canopy (Thom 1971; Jackson 1981; Parlange and Brutsaert 1989), it can thus be estimated in the following approach,

$$
d=\frac{\int_{0}^{h} z<u^{\prime} w^{\prime}>_{z} \mathrm{~d} z}{\int_{0}^{h}<u^{\prime} w^{\prime}>_{z} \mathrm{~d} z} \approx h-\frac{1}{u_{*}} \int_{0}^{h}<u^{\prime} w^{\prime}>\mathrm{d} z
$$

where the subscript $z$ represents the derivative with respect to $z$, and $u_{*}$ is the friction velocity at the canopy top, $u_{*}=\sqrt{-\left\langle\left(u^{\prime} w^{\prime}\right)_{h}\right\rangle}$. Based on Eq. 19, the zero-displacement heights, $d$, from the plant- and field-scale approaches are $0.71 h$ (PS-1), $0.72 h$ (PS-2), $0.81 h$ (FS-1), and $0.82 h$ (FS-2), respectively. The difference between the two 
Fig. 6 Energy spectra immediately above the canopy top $(z / h=1.1)(\mathbf{a}) E_{u u}$; (b) $E_{w w} ;(\mathbf{c}) E_{v v}$
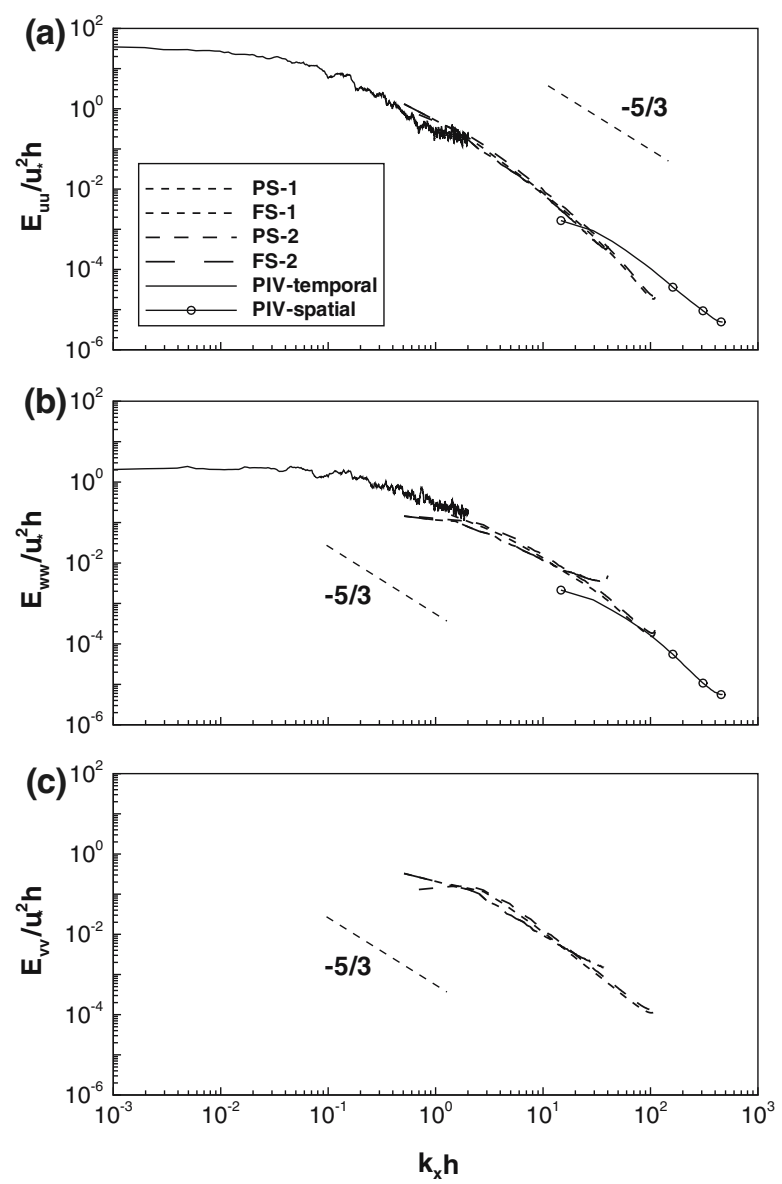

approaches is a result of the difference between canopy drag models. The field-scale approach tends to over-predict the canopy drag thus leading to a larger displacement height $d$. However, both approaches are in agreement with typical values of $d$ (ranging between $60 \%$ and $80 \%$ of the canopy height as quoted by Brutsaert (1982) and Kaimal and Finnigan (1994).

\subsection{Energy spectra}

Figure 6 shows the one-dimensional energy spectra of $u^{\prime}, v^{\prime}$, and $w^{\prime}, E_{u u}, E_{v v}$, and $E_{w w}$, with respect to the streamwise wavenumber, $k_{x}$, at $z / h=1.1$. The LES spectra are first spatially averaged in the spanwise direction and subsequently time averaged. The spectra of $u^{\prime}$ and $w^{\prime}$ from the PIV measurements are also plotted in the figures ( $E_{v v}$ is unavailable for the PIV measurements). Energy spectra were calculated from the PIV dataset by treating it as time series, invoking Taylor's frozen turbulence hypothesis, as well as by directly calculating the spatial energy spectra (see van Hout et al. (2007) for details). The latter ranges between $15<k_{x} h<5 \times 10^{2}$ while the PIV temporal spectra ranges between $1 \times 10^{-3}<k_{x} h<3$. The wavenumber range covered by the LES fills the gap between the PIV temporal and spatial spectra. 

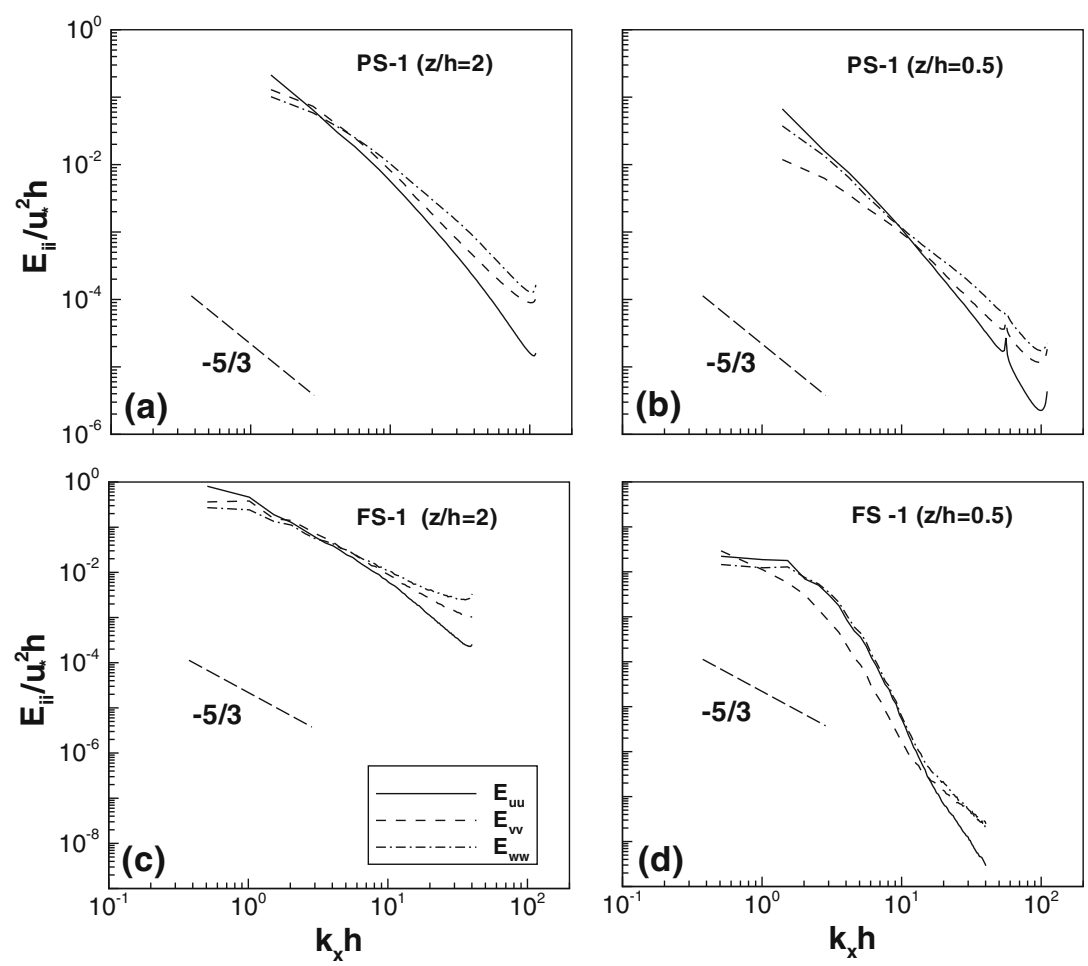

Fig. 7 Energy spectra above and within the canopy

The combination of spatial and temporal PIV measurements and LES allows us to couple a wide range of turbulence scales. Note that the profiles of PS- 1 and PS- 2 collapse, similarly FS-1 and FS-2 overlap. The plant- and field-scale approaches predict the same spectral slopes for the respective three components within the overlapping wavenumber range. The energy spectrum of the streamwise component, $E_{u u}$, has a faster roll-off rate than $-5 / 3$ within the high wavenumber region. The same trend was also observed in the Moga forest (Kaimal and Finnigan 1994). However, $E_{w w}$ and $E_{v v}$ approximately follow the $-5 / 3$ scope This spectral trend is similar to, but weaker than the trends observed inside the canopy (see Fig. 7b). This is likely because the canopy drag is more efficient at extracting energy from the $u$ component than from the $w$ and $v$ components considering that $u$ is much larger than $w$ and $v$ in magnitude (resulting in a quadratic drag in $u$ but linear in $w$ and $v$ in Eq. 12). Part of this energy is not immediately dissipated but converted into kinetic energy of wake eddies behind plant elements (leaves and stems), leading to a spectral shortcut or bypass process as discussed in (Finnigan 2000). Note, however, that in the high wavenumber portions of the PIV data, the roll-off trends are reversed: the $E_{w w}$ spectra decay faster than the $E_{u u}$ ones (van Hout et al. 2007). The present LES does not resolve down to these small scales, so we prefer not to speculate on possible reasons for the different trends.

Figure 7 shows the energy spectra farther above and at the middle of the canopy, $z / h=2$ and 0.5 , respectively, by the plant-scale approach (Fig. 7a, b) and the fieldscale approach (Fig. 7c, d). Only the results of PS-1 and FS-1 are shown here since they 

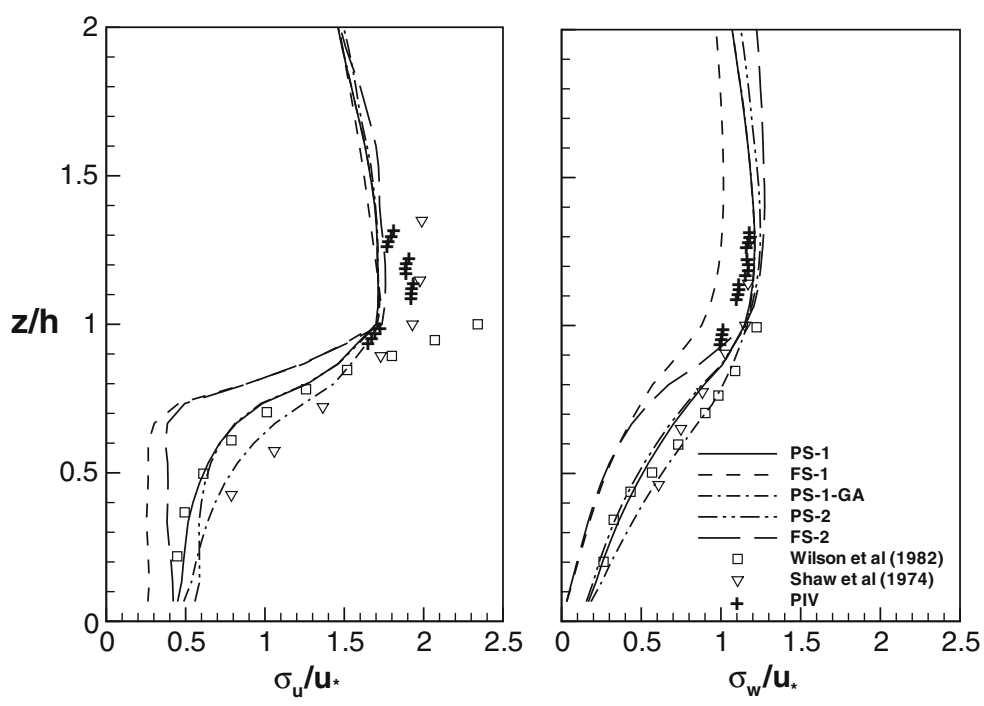

Fig. 8 Profiles of normalized rms velocities, $u_{*}$ is the friction velocity at the canopy top

collapse with those of PS-2 and FS-2, respectively. No PIV data are available at these elevations. In Fig. $7 \mathrm{a}, E_{w w} \approx 3 / 4 E_{u u}$ within $4<k_{x} h<10$, indicating a turbulence state close to local isotropy in the free airstream. The local isotropy above the canopy is also observed in Fig. 7c within $3<k_{x} h<9$ by FS-1. However, it is not observed deep inside the canopy (Fig. 7b, d). At the middle of the canopy $(z / h=0.5)$, PS- 1 shows that $E_{v v}$ and $E_{w w}$ have a slower roll-off rate than $E_{u u}$ for $k_{x} h>10$ (Fig. 7b). This is further evidence that the canopy drag extracts more kinetic energy from the streamwise velocity component to boost motions of wake-scale eddies. Figure 7d shows that FS-1 predicts a very rapid roll-off rate of the energy spectra inside the canopy, implying that this approach overestimates the canopy drag at these scales. The small peaks in Fig. $7 \mathrm{~b}$ (more appreciable in $E_{u u}$ ) results from the spatial periodicity of the imposed drag force in the flow field in PS-1.

\subsection{Velocity standard deviation and skewness}

Figure 8 shows the root-mean-square (rms) velocity profiles, e.g. $\sigma_{u}=\sqrt{<u^{\prime 2}}>$, normalized by the friction velocity at the canopy top, $u_{*}$. The experimental data of Shaw et al. (1974) and Wilson et al. (1982), and the present PIV measurements are also shown in the figure. Within the corn canopy, the predictions of $\sigma_{u} / u_{*}$ and $\sigma_{w} / u_{*}$ by the plant-scale approach (PS-1 and PS-2) agree with the experimental data of Wilson et al. (1982), while the field-scale approach (FS-1 and FS-2) underpredicts both $\sigma_{u} / u_{*}$ and $\sigma_{w} / u_{*}$. Near the ground, the result of FS-2 is closer to that of PS-1 than FS- 1 for $\sigma_{u}$. For $z / h<0.7, \sigma_{u}$ decreases quickly with depth and remains at a finite value of 0.5 very close to the ground. The spanwise component $\left(\sigma_{v}\right)$ shows the same features as $\sigma_{u}$ and is not shown here. The roll-off rate of $\sigma_{w}$ immediately below the canopy top is less steep than that of $\sigma_{u}$. This implies that the horizontal moments are damped more efficiently than the vertical ones by the canopy drag inside the canopy. At the canopy top, the field-scale approaches predict values of 1.7 and 1.2 for $\sigma_{u} / u_{*}$ and $\sigma_{w} / u_{*}$, 

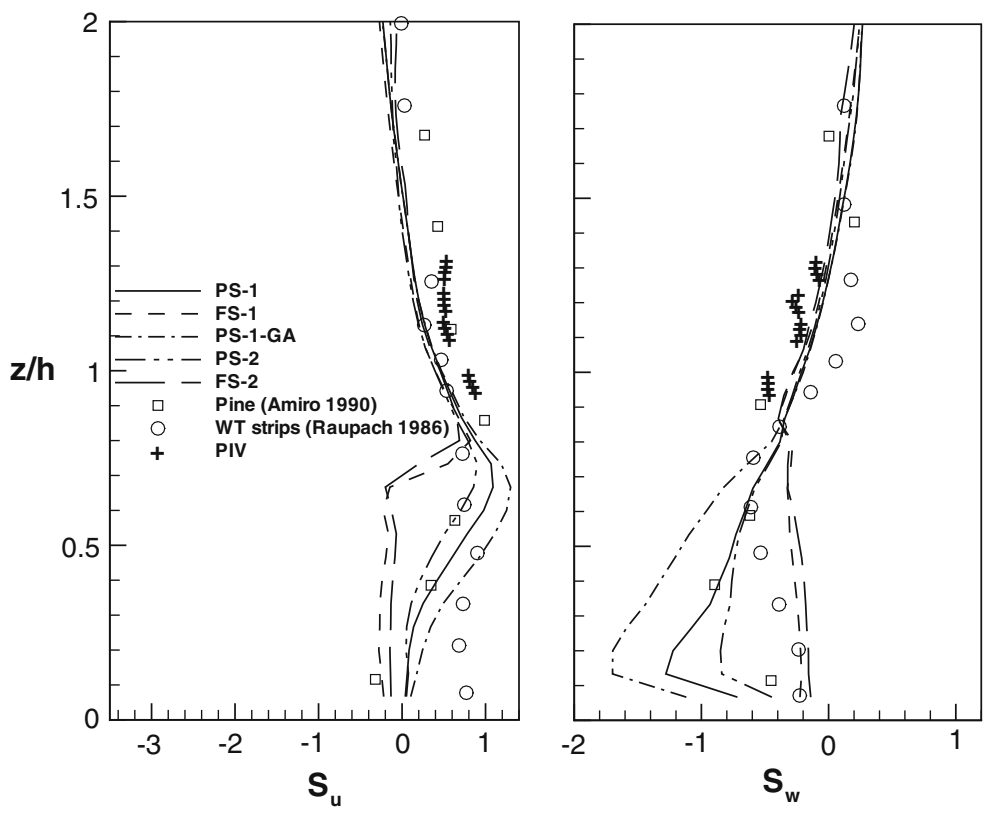

Fig. 9 Skewness factor of streamwise and vertical velocity components

respectively. FS-1 and FS-2 show the same value of 1.7 for $\sigma_{u}$. FS-1 shows a smaller $\sigma_{w}$ value of 1.0 while FS-2 predicts the same value as that by PS-1 and PS-2. The PIV data show, respectively, 1.8 and 1.1 , and they are 1.9 and 1.15 from the measurement of Shaw et al. (1974), whereas the data of Wilson et al. (1982) show a larger value of 2.3 for $\sigma_{u} / u_{*}$ and a consistent value of 1.2 for $\sigma_{w} / u_{*}$. The gap-averaged data (PS-1-GA) are $5-20 \%$ larger than the PS-1 data. Above the canopy, the numerical predictions of $\sigma_{u}$ are lower than the experimental data. The PS-1-GA profile collapses with the PS-1 profile above the canopy top, indicating the homogeneity of turbulence above the canopy.

Figure 9 shows the skewness of streamwise and vertical velocity components, e.g. $S_{u}=<u^{\prime 3}>/ \sigma_{u}^{3}$. Above the canopy, all numerical cases predict similar $S_{u}$ and $S_{w}$ profiles, and their agreement with the PIV measurements is fair. Since no data are available from Shaw et al. (1974) and Wilson et al. (1982), we plot the data from a pine forest (Amiro 1990) and in a wind-tunnel study (Raupach et al. 1986), for reference. The published data of velocity skewness shows considerable scatter inside the canopy (see Finnigan 2000), probably due to canopy morphological differences and insufficient sampling conditions to reach higher moment statistical convergence. There are also significant differences inside the canopy between the plant- and the field-scale predictions. The prediction by the plant-scale approach (PS-1 and PS-2) is close to the measurement data of the pine inside the canopy. Below $z / h=0.7$, FS- 1 predicts a nearly constant $S_{u}$ (weakly negative) and $S_{w}$. At $z / h=0.65$, FS-2 shows a negative peak of $S_{u}$ where PS-1 and PS-2 show positive peaks in agreement with experimental data. All simulation cases show negative $S_{w}$ inside the canopy, as do the experiments. The field-scale predictions of $S_{w}$ (FS-1 and FS-2) are only slightly negative while the plant-scale predictions (PS-1 and PS-2) are much larger and show peaks near the 
Fig. 10 Mean shear stress (sum of the resolved-scale and SGS components, $-\left(<u^{\prime} w^{\prime}>\right.$ $\left.\left.+<\tau_{x z}>\right)\right)$

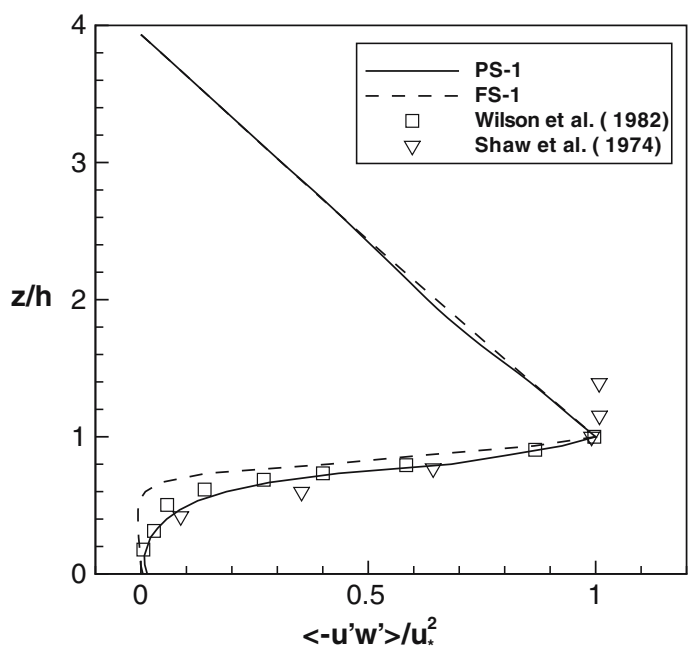

ground $(z / h=0.15)$. The positiveness of $S_{u}$ and negativeness of $S_{w}$ have been shown in many different canopies (Finnigan 2000), indicating strong downward momentum penetration into canopies.

\subsection{Vertical structure of momentum flux, correlation coefficient and Smagorinsky coefficient}

In Fig. 10 the vertical profiles of Reynolds shear stress (momentum flux), $-<u^{\prime} w^{\prime}>$, normalized by $u_{*}^{2}$ are plotted. The experimental data of Shaw et al. (1974) and Wilson et al. (1982) are also shown in the figure. Note that only the results of PS-1 and FS-1 are shown here since they are almost identical to those of PS-2 and FS-2, respectively. The PIV results are shown here because it is difficult to obtain accurate $u_{*}$ when the measurement stations are away from the canopy top. The momentum fluxes predicted from the present LES are the sum of resolved-scale Reynolds stress and the SGS component, $\left\langle\tau_{x z}>\right.$, where $\tau_{x z}$ is negligible except around the canopy top (not shown here).

Within the corn canopy, the prediction by PS-1 agrees with the experimental data better than that by FS-1. Above the canopy, both simulations show that the mean shear stress linearly decreases to the zero value imposed at the top boundary of the computational domain. A similar shear-stress profile was observed in the wind-tunnel experiment of Seginer et al. (1976), probably due to a finite value of pressure gradient existing in the windtunnel. Many field experiments have observed a constant stress layer above canopies, similar to the data of Shaw et al. (1974) shown in the figure. As in most previous large eddy simulations of canopy flows, the shear-free boundary condition imposed at the top boundary and the mean pressure gradient forcing leads to a linear mean momentum flux profile starting immediately above the canopy. This differs from the more nearly constant flux layer observed in the field. This discrepancy can only be remedied with a much larger but unaffordable computational domain, or by modifying the forcing schemes (e.g., Su et al. 1998). These options are outside the scope of the present paper. The expectation is that other turbulence statistics inside 
Fig. 11 Profile of correlation coefficient, $r_{u w}=-<u^{\prime} w^{\prime}>/$ $\sigma_{u} \sigma_{w}$

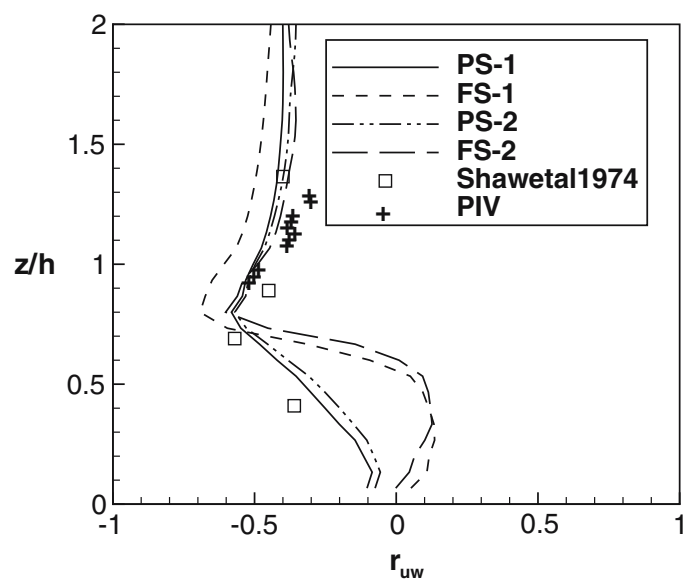

the canopy are not affected significantly by this difference in shear stress profile above the canopy.

The wall stress is negligible compared to the shear stress at the canopy top, implying that the horizontal momentum is mostly absorbed by the canopy. This fact also implies that the bottom boundary condition of the wall stress (imposed with the law-of-thewall) exerts little effect on turbulence structures above the canopy. The observation of the very weak momentum flux (Fig. 10) and the finite value of velocity variance of $\sigma_{u}$ (Fig. 8) near the ground suggests that there exists some kind of "sloshing" motion deep inside the canopy, which is inactive in transporting horizontal momentum, thus also called "inactive motion" by Bradshaw (1967).

Figure 11 shows the correlation coefficient, $r_{u w}=<u^{\prime} w^{\prime}>/ \sigma_{u} \sigma_{w}$, which is usually used to measure the efficiency of momentum transport. The results of FS-2 agree with those of FS-1 inside the canopy but match those of the plant-scale simulations above the canopy. The predictions by the plant-scale approach (PS-1 and PS-2) agree well with the experimental data of the present PIV and Shaw et al. (1974). At $z / h=0.8$, all cases predict peaks, -0.65 by FS- 1 and -0.55 in all other cases, consistent with the experimental data of -0.58 (Shaw et al. 1974). The peak point of $r_{u w}$ implies that turbulence is the most efficient in transporting momentum at this elevation. Below this elevation, $r_{u w}$ attenuates rapidly, another evidence of inactive motion deep inside the canopy. Well above the canopy top, $r_{u w}$ predicted by the plant-scale approach (PS-1 and PS-2) shows a nearly constant value, -0.4 .

Figure 12 shows the dynamic Smagorinsky coefficient $C_{S}$ calculated in terms of Eq. 6 and then averaged in time and horizontal planes. The predictions by FS- 2 has a similar pattern to that in Fig. 11, i.e., it follows FS-1 inside the canopy but the plant-scale cases above the canopy. Above the canopy top, $C_{S}$ has constant values of 0.23 for FS-1, but varies slightly about 0.16 for all other cases. The difference between FS-1 and FS-2 above the canopy may be due to the relative large grid spacings in FS-1 and that the scale-dependent lagrangian dynamic model may have some sensitivity to large grid size. This needs further investigations. PS-1 and PS-2 predict a small peak immediately above the canopy, but $C_{s}$ remains near 0.16 below the canopy (not near the ground). However, $C_{s}$ decreases sharply below the canopy top in FS-1 and FS-2. All the cases have a rapid decrease near the ground $(z / h<-0.15)$, demonstrating 
Fig. 12 Vertical profile of mean dynamic Smagorinsky coefficient $<C_{S}>$

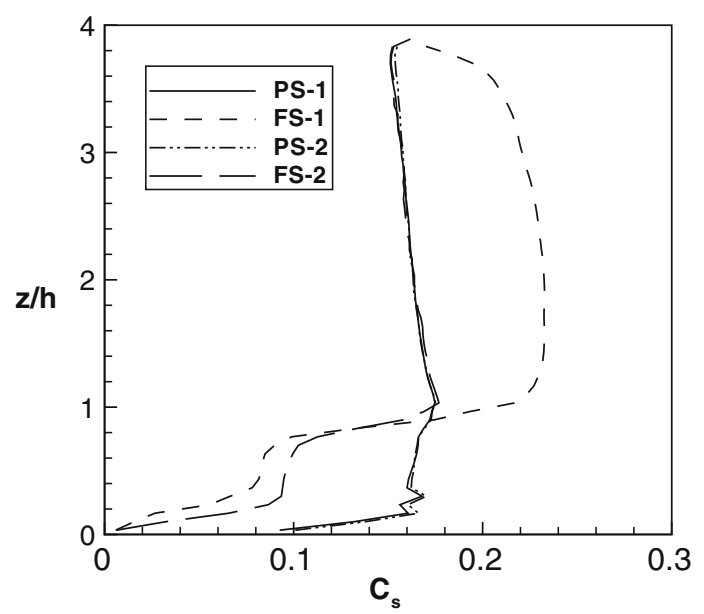

that the scale-dependent dynamic Lagrangian model predicts the near-wall damping effect.

\subsection{Instantaneous velocity fluctuation field}

Turbulent flows over canopies are characterized by intermittent sweep and ejection motions as demonstrated by many experimental studies. Figure 13 shows the instantaneous fluctuating velocity field $\left(u^{\prime \prime}\right.$ and $\left.w^{\prime \prime}\right)$ at two $x-z$ vertical planes of $j y=25$ (or $y / L_{y}=0.38$ ) and 37 (or $y / L_{y}=0.56$ ), where $j y$ is the grid index in the $y$ direction. Only the results from PS-1 and FS-1 are shown here, since the observed patterns of turbulent structures are similar to those of PS-2 and FS-2, respectively. In PS-1, these two planes lie in the middle of the wide and narrow gaps between two neighbouring plant rows, respectively (as shown in Fig. 13e). Figure 13a (PS-1) shows strong Q4 (sweep) events, which are weakened by the closely surrounding plants as shown in Fig. 13c (PS-1), indicating, not surprisingly, that the downward gusts penetrate more easily into the wide space between the canopy plants. Q2 (ejection) events are shown in Fig. 13c, but are of smaller magnitude than the Q4 events in Fig. 13a. Figure 13b, d (FS-1) also show sweep and ejection events around the canopy top. The most prominent phenomenon shown in Fig. 13 is that the plantscale approach predicts the deep penetration of the gust into the canopy (Fig. 13a) which is not observed in the field-scale approach. This is additional evidence that the field-scale approach is a less accurate method to predict turbulence structures inside the canopy. The ejections and sweeps are the important mechanism of momentum transfer. Figure 13 shows that the momentum transfer between atmosphere and canopy occurs mostly around the canopy top. Different from a turbulent wall boundary layer where ejections contribute most to the momentum flux (above the buffer layer) (Lu and Willmarth 1973), Fig. 13 and many other instantaneous fluctuating velocity field views (not shown here) suggest that sweeps are the dominant contributor to the momentum flux inside the canopy. Turbulence in the plant canopy simulated by the plant-scale approach is further studied in a detailed quadrant-hole analysis of canopy turbulence in a followup paper (Yue et al. 2007). Finally, we remark that some initial simulations using plant-scale representations but with the wind direction shifted by 


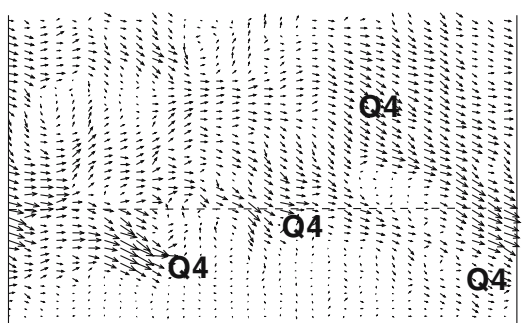

(a) PS-1 vertical plane $\mathrm{jy}=\mathbf{2 5}$

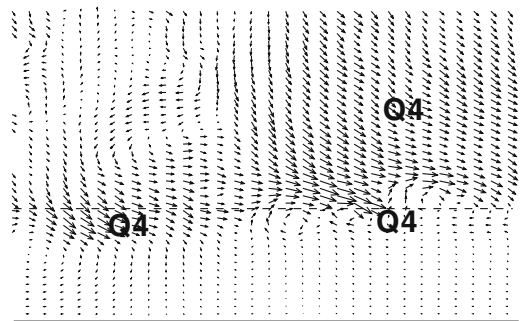

(b) FS-1 vertical plane jy $=25$

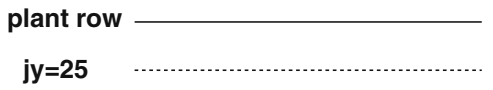

plant row

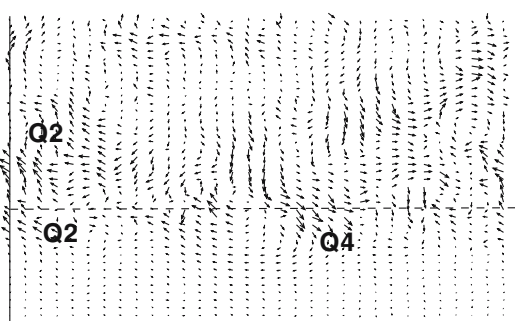

(c) PS-1 vertical plane $\mathrm{jy}=37$

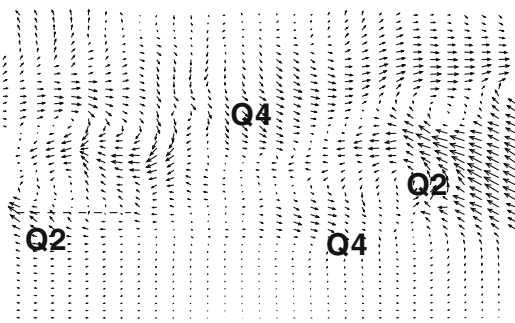

(d) FS-1 vertical plane jy $=37$

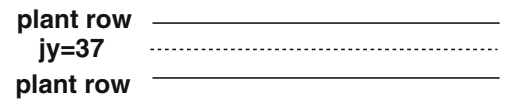

(e)schematic of locations of extracted planes

Fig. 13 Instantaneous fluctuating velocity at two $x z$ vertical planes with $j y=25$ (or $y / L_{y}=0.38$ ) ((a) for plant-scale and (b) for field-scale) and 37 (or $\left.y / L_{y}=0.56\right)((\mathbf{c})$ for plant-scale and (d) for field-scale). The dashed line represents the top of the canopy. (e) Schematic of locations of extracted planes for the plant-scale case

$30^{\circ}$ from the plant row direction have also been performed. Preliminary results (not shown) indicate that the depth of penetration of turbulence into the canopy is still more pronounced in this case than in the field-scale simulations, in which the canopy structure has no preferred direction.

\section{Summary and Conclusions}

Large-eddy simulations of turbulent flows over a corn canopy have been performed to investigate how to best represent the plant architecture and spatial arrangement. A new numerical representation of canopies, termed as "plant-scale" (PS) approach, is presented in this paper. The PS approach takes into account the heterogeneity of the canopy field, and the approximate features of individual plants are resolved. Numerical simulations based on the traditional "field-scale" (FS) approach are also conducted for comparison. To test the sensitivity of the numerical models on the computational domain size and the grid resolution, four simulation cases have been conducted. The computational LES results are compared with those of previous field experiments and recent PIV field measurements. The mean velocity and mean shear stress predicted by the FS approach are generally comparable to those obtained by the 
PS approach and to the experimental data. Conversely, the FS-approach's predictions of rms velocity, streamwise velocity skewness, and correlation coefficient are not as good as those of the PS approach based on comparison with the experimental data. In general, we can conclude that the plant-scale approach shows better performance in prediction of canopy turbulence than the field-scale approach. The predictions of energy spectra by both the FS and PS approaches show excellent agreement with the field experimental data available at various wavenumber ranges near the canopy height. Inside the canopy, the FS spectra show more rapid roll-off near the cutoff wave-number as compared to the PS results.

The mean velocity profiles show maximum shear around the canopy top, providing the main production of canopy turbulence. The present numerical models predict a displacement height consistent with the typical range observed in other canopies. The slight differences may be attributed to the high leaf area index of the present corn field, although the differences are within the measurement uncertainty. The analysis of energy spectra within and above the canopy indicates that the canopy drag extracts more kinetic energy from the $u^{\prime}$ component and converts it into motions of wake-scale eddies behind plant elements. The combination of LES and the PIV temporal and spatial measurements provides a wide scale range of turbulence structures.

The prediction of the velocity variances by the plant-scale approach shows good agreement with the experimental data within and above the canopy. The plant-scale approach also yields a good agreement of the vertical profiles of the mean momentum flux with the experimental data. The mean momentum flux decreases almost exponentially within the canopy and linearly above the canopy. The observation of a very weak shear stress and a finite value of velocity variance $\sigma_{u}$ near the ground suggests the existence of inactive motions deep inside the canopy. All the simulations predict peaks of correlation coefficient at the same location within the canopy $(z / h=0.8)$, indicating that the most efficient location of transporting momentum by turbulence is a bit below the canopy top. The fluctuating instantaneous velocity fields visualize the turbulence sweep and ejections events, suggesting that the sweeps are the major contributor to the momentum flux. The field-scale results show excessive damping of deep penetrating gusts into the canopy, suggesting that the plant-scale approach is a more appropriate method to investigate turbulence structures within the canopy.

Acknowledgements This study was partially supported by the National Science Foundation Biocomplexity program under Grant No. BES-0119903.

\section{References}

Albertson JD, Parlange MB (1999a) Natural integration of scalar fluxes from complex terrain. Adv Water Resour 23:239-252

Albertson JD, Parlange MB (1999b) Surface length-scales and shear stress: implications for landatmosphere interaction over complex terrain. Water Resour Res 35:2121-2132

Amiro BD (1990) Drag coefficients and turbulence spectra within three boreal forest canopies. Boundary-Layer Meteorol 52:227-246

Bou-Zeid E, Meneveau C, Parlange MB (2005) A scale-dependent Lagrangian dynamic model for large eddy simulation of complex turbulent flows. Phys Fluids 17:025105

Bradley EF, Finnigan JJ (1973) Heat and mass transfer in the plant-air continuum. Proc first Australasian heat and mass conference, Monash University, Melbourne, Australia, pp 57-76

Bradshaw P (1967) 'Inactive' motion and pressure fluctuations in turbulent boundary layers. J Fluid Mech 30:241-258 
Brunet Y, Finnigan JJ, Raupach MR (1994) A wind tunnel study of air flow in waving wheat: singlepoint velocity statistics. Boundary-Layer Meteorol. 70:95-132

Brutsaert W (1982) Evaporation into the atmosphere: theory, history and applications. D. Redel Pub. Co., Dordrecht, 299 pp

Dwyer MJ, Patton EG, Shaw RH (1997) Turbulent kinetic energy budgets from a large-eddy simulation of airflow above and within a forest canopy. Boundary-Layer Meteorol. 84:23-43

Finnigan JJ (1979) Turbulence in waving wheat I. Mean statistics and honami. Boundary-Layer Meteorol. 16:181-211

Finnigan J (2000) Turbulence in plant canopies. Annu Rev Fluid Mech 32:519-571

Fitzmaurice L, Shaw RH, Paw UKT, Patton EG (2004) Three-dimensional scalar microfront system in a large-eddy simulation of vegetation canopy flow. Boundary-Layer Meteorol. 112:107-127

Gao W, Shaw RH, Paw UKT (1989) Observation of organized structur in turbulent flow within and above a forest canopy. Boundary-Layer Meteorol 47:349-377

Gardiner BA (1994) Wind and wind forces in a plantation spruce forest. Boundary-Layer Meteorol 67:161-186

Germano M, Piomelli U, Moin P, Cabot W (1991) A dynamic subgrid-scale eddy viscosity model. Phys Fluids A 3:1760-1765

Jackson PS (1981) On the displacement height in the logarithmic velocity profile. J Fluid Mech 111:15-25

Kaimal JC, Finnigan JJ (1994) Atmospheric boundary layer flows: their structure and measurement. Oxford University Press, New York

Kanda M, Hino M (1994) Organized structures in developing turbulent flow within and above a plant canopy using a large eddy simulation. Boundary-Layer Meteorol 68:237-257

Lilly DK (1992) A proposed modification of the Germano subgrid-scale closure method. Phys Fluids A $4: 633-635$

Lu SS, Willmarth WW (1973) Measurements of the structure of the Reynolds stress in a turbulent boundary layer. J Fluid Mech 60:481-511

Meneveau C, Lund T, Cabot W (1996) A Lagrangian dynamic subgrid-scale model of turbulence. J Fluid Mech 319:353-385

Meneveau C, Katz J (2000) Sale-invariance and turbulence models for large-eddy simulation. Annu Rev Fluid Mech 32:13-32

Moeng C-H (1984) A large-eddy simulation model for the study of planetary boundary-layer turbulence. J Atmos Sci 41:2052-2062

Orszag SS (1970) Transform method for calculation of vector coupled sums: application to the spectral form of vorticity equation. J Atmos Sci 27:890-895

Parlange MB, Brutsaert W (1989) Regional roughness of the lands forest and surface shear stress under neutral conditions. Boundary-Layer Meteorol 48:69-81

Patton EG, Shaw RH, Judd MJ, Raupach MR (1998) Large-eddy simulation of windbreak flow. Boundary-Layer Meteorol. 87:275-306

Porté-Agel F, Meneveau C, Parlange MB (2000) A scale-dependent dynamic model for large-eddy simulation: application to a neutral atmospheric boundary layer. J Fluid Mech 415:261-284

Raupach MR, Thom AS (1981) Turbulence in and above plant canopies. Annu Rev Fluid Mech 13:97-129

Raupach MR, Coppin PA, Legg BJ (1986) Experimentas on scalar dispersion within a model plant canopy. Part I: the turbulence structure. Boundary-Layer Meteorol 35:21-52

Seginer I, Mulhearn PJ, Bradley EF, Finnigan JJ (1976) Turbulent flow in a model plant canopy. Boundary-Layer Meteorol 10:423-453

Shaw RH, Den Hartog G, Neumann HH (1988) Influence of foliar density and thermal stability on profiles of Reynolds stress and turbulence intensity in a deciduous forest. Boundary-Layer Meteorol 45:391-409

Shaw RH, Patton EG (2003) Canopy element influences on resolved- and subgrid scale energy within a large-eddy simulation. Agric For Meteorol 115:5-17

Shaw RH, Schumann U (1992) Large-eddy simulation of turbulent flow above and within a forest. Boundary-Layer Meteorol 61:47-64

Shaw RH, Silversides RH, Thurtell GW (1974) Some observations of turbulence and turbulent transport within and above plant canopies. Boundary-Layer Meteorol 5:429-449

Shen S, Leclerc MY (1997) Modeling the turbulence structure in the canopy layer. Agric For Meteorol $87: 3-25$ 
Su H-B, Shaw RH, Paw UKT, Moeng C-H, Sullivan PP (1998) Turbulent statistics of neutrally stratified flow within and above a sparse forest from large-eddy simulation and field observations. Boundary-Layer Meteorol 88:363-397

Thom AS (1971) Momentum absorption by vegetation. Quart J Roy Meteorol Soc 97:414-428

van Hout R, Zhu W, Luznik L, Katz J, Kleissl J, Parlange MB (2007) PIV measurements in the atmospheric boundary layer within and above a mature corn canopy. Part A: statistics and energy flux. J Atmos Sci (in press)

Watanabe T (2004) Large-eddy simulation of coherent turbulence structures associated with scalar ramps over plant canopies. Boundary-Layer Meteorol 112:307-341

Wilson JD, Ward DP, Thurtell GW, Kidd GE (1982) Statistics of atmospheric turbulence within and above a corn canopy. Boundary-Layer Meteorol 24:495-519

Wolfenbarger LL, Phifer PR (2000) The ecological risks and benefits of genetically engineered plants. Science 290:2088-2093

Yue W, Meneveau C, Parlange MB, Zhu W, van Hout R, Katz J (2007) A comparative quadrant analysis of turbulence in a plant canopy. Water Resour Res (in press)

Zhu W, van Hout R, Luznik L, Kang HS, Katz J, Meneveau C (2006) A comparison of PIV mesurements of canopy turbulence performed in the field and in a wind tunnel model. Exp Fluids 41:309-318 